\title{
Electric Assumptions for Piezoelectric Laminate Analysis
}

\author{
K.Y.Sze* , X.-M.Yang \\ Department of Mechanical Engineering, The University of Hong Kong, \\ Pokfulam Road, Hong Kong SAR, P.R.CHINA.
}

\section{H.Fan}

School of Mechanical \& Production Engineering, Nanyang Technological University, Nanyang Avenue, SINGAPORE 639798.

\begin{abstract}
In computational analysis of piezoelectric laminates which may comprise piezoelectric and nonpiezoelectric plies, the transverse electric field is often assumed to be piecewise constant along the transverse direction. This conventional assumption may lead to significant errors unless the piezoelectric ply is considerably thinner than the overall thickness of the laminate. To this end, two alternate assumptions termed EL and DC on the spatial distributions of the electric variables are proposed. In EL, the transverse electric field is taken to be piecewise linear along the transverse direction. In the DC assumption, the transverse electric displacement is taken to be piecewise constant along the transverse direction. The rationales leading to the proposed assumptions are discussed. Numerical examples indicate that the proposed assumptions are markedly more accurate than the conventional one. However, EL becomes inaccurate when its piecewise function spans more than one ply. In this light, DC often consumes less electric potential d.o.f.s and is more efficient than EL.
\end{abstract}

Keywords: piezoelectric, laminate, smart structure, computation

* corresponding author, email address : kysze@hku.hk 


\section{INTRODUCTION}

Piezoelectric laminates comprise perfectly bonded piezoelectric and, probably, non-piezoelectric (conducting-elastic and dielectric-elastic) plies. Typical examples of piezoelectric laminates are bimorphs and "smart" plates fabricated for shape and vibration control. Like most engineering problems, analytical solutions for piezoelectric laminates are limited to simplistic cases. To this end, various lamination models suitable for computational implementation have been proposed. The most natural and straight forward approach is the one based on the fully layerwise formulation in which all the primary variables, namely, the displacement and electric potential, are piecewisely approximated within each ply and their continuities are maintained at $\mathrm{C}^{0}$ at the ply-to-ply interfaces (Ha et al 1992; Heyliger et al 1994; Tzou et al 1994; Tzou \& Ye 1996; Sze \& Pan 1999; Sze et al 2000). Its drawback, however, is that the computational cost increases rapidly with the number of plies.

In the analysis of conventional or non-piezoelectric laminates, it is popular for the sake of reducing computational cost that various plate theories with (smooth) through-thickness variation of the displacement are employed (Lo et al 1977; Reddy 1984; Noor \& Burton 1989; Sze et al 2000). Following the same philosophy, it is often seen in the analysis of piezoelectric laminates that through-thickness approximation is adopted for some primary variables whereas layerwise approximation is retained for the others. In this context, Heyliger et al (1994) and Trindade et al (2001) employed the through-thickness approximation for the transverse deflection whereas the inplane displacement and the electric potential are layerwisely approximated. Chee et al (2000) employed the second and third orders through-thickness approximations for the transverse and inplane displacements, respectively. The electric potential is layerwisely approximated. Almajid et al (2001) and Sheikh et al (2001) adopted respectively the Kirchhoff plate theory and the first order Mindlin plate theory for approximating the displacement. Again, the layerwise approximation is adopted for the electric potential. To further reduce the computational load, some researchers even disregard the in-plane electric field components in computing the electromechanical energy (Lammering 1991, Trindade et al 2001).

A note-worthily observation common to the afore-reviewed work is that the electric potential is assumed to be piecewise linear along the transverse direction. The assumption leads to a piecewise Constant transverse Electric field component along the transverse direction and, thus, is abbreviated as $E C$. As the transverse electric field is discontinuous at the interfaces of dissimilar plies and cannot be reasonably mimicked by through-thickness approximations of the electric potential, the assumption appears to be natural and computationally most efficient. Unfortunately, it may lead to significant errors unless the piezoelectric ply is considerably thinner than the overall thickness of 
the laminate. On the other hand, very few researchers have employed nonlinear layerwise approximations for the electric potential (Yang 1999; Fernandes \& Pouget 2001).

In this paper, two electric assumptions on the spatial variations of the electric variables are proposed. The first one is straight forward and is obtained by enhancing EC with quadratic variation of the electric potential along the transverse direction. It leads to a piecewise Linear transverse Electric field along the transverse direction and, thus, is abbreviated as EL. The second assumption, abbreviated as $D C$, assumes the transverse electric Displacement to be piecewise Constant along the transverse direction within the same electric stack. The latter is defined as a block of nonconducting plies and ply-to-ply interfaces sandwiched by a pair of electric gauge levels over which the electrode potential is either prescribed or tapped. The rationales leading to the proposed assumptions are discussed. After combining with the Mindlin plate theory, various electric assumptions are put into computational trials. When the piecewise function span only one ply, the proposed assumptions are close in accuracy and are more accurate than EC. However, EL becomes inaccurate when its piecewise function spans more than one ply. As an electric stack can comprise several plies, DC often consumes less electric potential d.o.f.s and can be significantly more efficient than EL.

\section{Kinematics of the Mindlin Plate Theory}

The displacement approximation of the Mindlin plate theory is through-thickness and can be expressed as: $u=u_{o}+z u_{1}, v=v_{o}+z v_{1}$ and $w=w_{o}$ in which $z$ is the transverse coordinate; $u_{i}$ 's, $v_{i}$ 's and $w_{0}$ are functions of the in-plane coordinates $x$ and $y$ only. The above displacement yields the following vectors of strain components:

$$
\boldsymbol{\epsilon}=\left\{\begin{array}{c}
\varepsilon_{x} \\
\varepsilon_{y} \\
\gamma_{x y}
\end{array}\right\}=\boldsymbol{\epsilon}_{o}+z \boldsymbol{\epsilon}_{1}=\left\{\begin{array}{c}
u_{o},_{x} \\
v_{o},{ }_{y} \\
u_{o},{ }_{y}+v_{o}, x
\end{array}\right\}+z\left\{\begin{array}{c}
u_{1},_{x} \\
v_{1},{ }_{y} \\
u_{1},{ }_{y}+v_{1}, x_{x}
\end{array}\right\}, \boldsymbol{\gamma}=\left\{\begin{array}{c}
\gamma_{z x} \\
\gamma_{y z}
\end{array}\right\}=\left\{\begin{array}{c}
w_{o},{ }_{x}+u_{1} \\
w_{o}, v_{y}+v_{1}
\end{array}\right\}
$$

where $\boldsymbol{\epsilon}_{i}$ 's and $\boldsymbol{\gamma}$ are independent of $z$. The transverse normal stress and strain components are dropped from the constitutive relation by assuming the plane stress condition along the z-direction.

\section{Constitutive Relation and Electromechanical Energy}

The functional requirement of piezoelectric laminates often, if not always, requires the piezoelectric 
plies to be poled along the transverse direction. With the poling direction along the $\mathrm{z}$-axis and the remaining material principal directions aligned with the $\mathrm{x}$ - and $\mathrm{y}$-axes, the following form of constitutive rule covers a wide range of piezoelectrics:

$$
\left\{\begin{array}{l}
\sigma_{x} \\
\sigma_{y} \\
\sigma_{z} \\
\tau_{y z} \\
\tau_{z x} \\
\tau_{x y} \\
D_{x} \\
D_{y} \\
D_{z}
\end{array}\right\}=\left[\begin{array}{ccccccccc}
c_{11} & c_{12} & c_{13} & 0 & 0 & 0 & 0 & 0 & e_{31} \\
c_{12} & c_{22} & c_{23} & 0 & 0 & 0 & 0 & 0 & e_{32} \\
c_{13} & c_{23} & c_{33} & 0 & 0 & 0 & 0 & 0 & e_{33} \\
0 & 0 & 0 & c_{44} & 0 & 0 & 0 & e_{24} & 0 \\
0 & 0 & 0 & 0 & c_{55} & 0 & e_{15} & 0 & 0 \\
0 & 0 & 0 & 0 & 0 & c_{66} & 0 & 0 & 0 \\
0 & 0 & 0 & 0 & e_{15} & 0 & -\kappa_{11} & 0 & 0 \\
0 & 0 & 0 & e_{24} & 0 & 0 & 0 & -\kappa_{22} & 0 \\
e_{31} & e_{32} & e_{33} & 0 & 0 & 0 & 0 & 0 & -\kappa_{33}
\end{array}\right]\left\{\begin{array}{c}
\varepsilon_{x} \\
\varepsilon_{y} \\
\varepsilon_{z} \\
\gamma_{y z} \\
\gamma_{z x} \\
\gamma_{x y} \\
\phi,_{x} \\
\phi,_{y} \\
\phi,_{z}
\end{array}\right\}
$$

where $\sigma$ 's, $\tau$ 's, D's and $\phi$ denote respectively the normal stress components, shear stress components, electric displacement components and the electric potential. Moreover, $c$ 's, $e$ 's and $\kappa$ 's are respectively the elastic, piezoelectric and permittivity coefficients. By incorporating the plane stress condition $\left(\sigma_{z}=0\right)$ along the transverse direction, (2) reduces to:

$$
\left\{\begin{array}{c}
\boldsymbol{\sigma} \\
D_{z}
\end{array}\right\}=\left[\begin{array}{cc}
\mathbf{C}_{=} & \mathbf{C}_{\times} \\
\mathbf{C}_{\times}^{T} & C_{\|}
\end{array}\right]\left\{\begin{array}{c}
\boldsymbol{\epsilon} \\
\phi,
\end{array}\right\},\left\{\begin{array}{c}
\boldsymbol{\tau} \\
\mathbf{D}_{L}
\end{array}\right\}=\left[\begin{array}{cc}
\mathbf{c}_{t} & \mathbf{e}_{t} \\
\mathbf{e}_{t}^{T} & -\boldsymbol{\kappa}_{t}
\end{array}\right]\left\{\begin{array}{c}
\boldsymbol{\gamma} \\
\boldsymbol{\phi}_{L}
\end{array}\right\}
$$

where

$$
\begin{aligned}
& \boldsymbol{\sigma}=\left\{\begin{array}{l}
\sigma_{x} \\
\sigma_{y} \\
\tau_{x y}
\end{array}\right\}, \boldsymbol{\epsilon}=\left\{\begin{array}{l}
\varepsilon_{x} \\
\varepsilon_{y} \\
\gamma_{x y}
\end{array}\right\}, \mathbf{C}_{=}=\left[\begin{array}{ccc}
c_{11}-c_{13}^{2} / c_{33} & c_{12}-c_{13} c_{23} / c_{33} & 0 \\
c_{12}-c_{13} c_{23} / c_{33} & c_{22}-c_{23}^{2} / c_{33} & 0 \\
0 & 0 & c_{66}
\end{array}\right], \\
& \mathbf{C}_{\times}=\left[\begin{array}{c}
e_{31}-c_{13} e_{33} / c_{33} \\
e_{32}-c_{13} e_{33} / c_{33} \\
0
\end{array}\right], C_{\|}=-\kappa_{33}-e_{33}^{2} / c_{33}, \boldsymbol{\tau}=\left\{\begin{array}{l}
\tau_{y z} \\
\tau_{z x}
\end{array}\right\}, \mathbf{D}_{L}=\left\{\begin{array}{l}
D_{x} \\
D_{y}
\end{array}\right\}, \boldsymbol{\phi}_{L}=\left\{\begin{array}{l}
\phi,_{x} \\
\phi_{y}
\end{array}\right\}, \\
& \mathbf{c}_{t}=\left[\begin{array}{cc}
c_{44} & 0 \\
0 & c_{55}
\end{array}\right], \mathbf{e}_{t}=\left[\begin{array}{cc}
0 & e_{24} \\
e_{15} & 0
\end{array}\right], \boldsymbol{\kappa}_{t}=\left[\begin{array}{cc}
\kappa_{11} & 0 \\
0 & \kappa_{22}
\end{array}\right] .
\end{aligned}
$$

Hence, the electromechanical energy $U$ per unit planar area of the laminated plate defined by $z \in\left[z_{0}\right.$, $z_{n}$ ] can be expressed as:

$$
\frac{\partial U}{\partial A}=\frac{1}{2} \int_{z_{0}}^{z_{n}}\left(\left\{\begin{array}{c}
\boldsymbol{\epsilon} \\
\phi,_{z}
\end{array}\right\}^{T}\left[\begin{array}{cc}
\mathbf{C}_{=} & \mathbf{C}_{\times} \\
\mathbf{C}_{\times}^{T} & C_{\|}
\end{array}\right]\left\{\begin{array}{c}
\boldsymbol{\epsilon} \\
\phi_{y_{z}}
\end{array}\right\}+\left\{\begin{array}{c}
\boldsymbol{\gamma} \\
\boldsymbol{\phi}_{L}
\end{array}\right\}^{T}\left[\begin{array}{cc}
\mathbf{c}_{t} & \mathbf{e}_{t} \\
\mathbf{e}_{t}^{T} & -\boldsymbol{\kappa}_{t}
\end{array}\right]\left\{\begin{array}{c}
\boldsymbol{\gamma} \\
\boldsymbol{\phi}_{L}
\end{array}\right\}\right) d z
$$

in which $A$ denotes the planar area. 


\section{Piecewise Constant Transverse Electric Field (EC) Assumption}

Figure 1 shows a corner of a laminated piezoelectric plate. Electric gauge levels at which the electric potential are either tapped or prescribed are defined at $z=z_{0}, z_{1}, \ldots, z_{n}$. Using the conventional piecewise linear assumption of the electric potential along the transverse direction, the electric potential between $z=z_{j-1}$ and $z_{j}$ is interpolated as:

$$
\phi^{j}=\phi^{j}(x, y, z)=N_{j}^{j} \phi_{j}+N_{j-1}^{j} \phi_{j-1}
$$

and thus,

$$
\phi=\phi(x, y, z)=\sum_{j=1}^{n} \Lambda_{j} \phi^{j}=\sum_{j=1}^{n} \Lambda_{j}\left(N_{j}^{j} \phi_{j}+N_{j-1}^{j} \phi_{j-1}\right)
$$

where

$$
\begin{aligned}
& N_{j}^{j}=\frac{z-z_{j-1}}{h_{j}}, N_{j-1}^{j}=\frac{z_{j}-z}{h_{j}}, \Lambda_{j}=\left\{\begin{array}{ll}
1 & \text { for } z \in\left[z_{j-1}, z_{j}\right] \\
0 & \text { otherwise }
\end{array}, h_{j}=z_{j}-z_{j-1},\right. \\
& \phi_{j}=\phi_{j}(x, y) \text { and } \phi_{j-1}=\phi_{j-1}(x, y) \text { are respectively the electric potential at } \mathrm{z}=z_{j} \text { and } \mathrm{z}=z_{j-1} .
\end{aligned}
$$

By substituting (1) and (6) into (4), the problem can then be computationally pursued by the electromechanical virtual work principle (EerNisse 1967; Allik \& Hughes 1970). As the core assumption here is a piecewise Constant transverse Electric field, it will be termed as $E C$.

\section{Piecewise Linear Transverse Electric Field (EL) Assumption}

Let $L$ denote the nominal side length of the plate, the ratio of the spatial derivatives of the electric potential can be estimated as:

$$
\phi,_{x}: \phi,_{x}: \phi,_{z} \approx \phi / L: \phi / L: \phi / h=h: h: L
$$

It is also known for shear deformable plates that

$$
\varepsilon_{x}: \varepsilon_{y}: \varepsilon_{z}: \gamma_{z x}: \gamma_{y z} \approx L: L: L: h: h
$$

From (2), (7) and (8),

$$
\begin{aligned}
D_{x}: D_{y} & : D_{z}=e_{15} \gamma_{z x}-\kappa_{11} \phi,_{x}: e_{24} \gamma_{y z}-\kappa_{22} \phi,,_{y}: e_{31} \varepsilon_{x}+e_{32} \varepsilon_{y}+e_{33} \varepsilon_{z}-\kappa_{33} \phi,_{z} \\
& \approx\left(e_{15} \varepsilon-\kappa_{11} \phi,_{z}\right) h:\left(e_{24} \varepsilon-\kappa_{22} \phi,_{z}\right) h:\left(e_{31} \varepsilon+e_{32} \varepsilon+e_{33} \varepsilon-\kappa_{33} \phi,_{z}\right) L \approx h: h: L
\end{aligned}
$$

where $\varepsilon \approx \varepsilon_{x} \approx \varepsilon_{y} \approx \varepsilon_{z}$. On the other hand, the fundamental deformation modes of plates are the stretching and flexural modes in which the in-plane strains $\varepsilon_{x}, \varepsilon_{y}$ and $\gamma_{x y}$ are linear functions of $z$. Hence, and also from (9), the pointwise satisfaction of the charge conservation equation: 


$$
D_{x},_{x}+D_{y},_{y}+D_{z},_{z}=0
$$

requires the electric potential to be a quadratic function of $z$. Thus, the piecewise linear assumption of electric potential in (6) is prone to error. To this end, the linear interpolation in (6) is enhanced with the quadratic term $4 N_{j}^{j} N_{j-1}^{j}$ and becomes:

$$
\phi=\phi(x, y, z)=\sum_{j=1}^{n} \Lambda_{j} \phi^{j}=\sum_{j=1}^{n} \Lambda_{j}\left(N_{j}^{j} \phi_{j}+4 N_{j}^{j} N_{j-1}^{j} \phi_{b}^{j}+N_{j-1}^{j} \phi_{j-1}\right)
$$

The quadratic term $N_{j}^{j} N_{j-1}^{j}$ vanishes at $z_{j}$ and $z_{j-1}$ such that $\phi_{j}$ and $\phi_{j-1}$ still represent the electric potentials at $z_{j}$ and $z_{j-1}$, respectively. By substituting (1) and (11) into (4), an expression on the electromechanical energy per unit planar area can be obtained. Again, the problem can be computationally pursued by the electromechanical virtual work principle. As the transverse Electric field along the transverse direction is piecewise Linear, the present assumption is termed as $E L$.

\section{Piecewise Constant Transverse Electric Displacement (DC) Assumption}

Further to the ratio among different electric displacement components given in (9), one can also get:

$$
D_{x},_{x}: D_{y},_{y}: D_{z},_{z} \approx D_{x} / L: D_{y} / L: D_{z} / h \approx h^{2}: h^{2}: L^{2}
$$

The above expression suggests the following approximation to (10):

$$
D_{z}, z=0
$$

for $h^{2} / L^{2}>>0$ or, equivalently, moderately thick plates. In other words, the transverse electric displacement is approximately piecewise constant along the transverse direction. Within an electric stack (defined as a block of non-conducting plies and ply-to-ply interface sandwiched by a pair of electric gauge levels over which the electrode potential is either prescribed or tapped), the continuity of the electric charge area density at ply-to-ply interfaces implies that $D_{z}$ is $\mathrm{C}^{0}$ at the interfaces. Consequently, $D_{z}$ is approximately constant along the transverse direction within the same electric stack. To realize (13) in the electromechanical energy calculation, a piecewise constant $D_{\mathrm{z}}$ is first assumed:

$$
D_{z}(x, y, z)=\sum_{j=1}^{n} \Lambda_{j}(z) D_{j}(x, y)
$$


in which $\Lambda$ has been defined in (6). By changing equation objects, the first relation in (3) is rewritten as:

$$
\left\{\begin{array}{c}
\boldsymbol{\sigma} \\
\phi,_{z}
\end{array}\right\}=\left[\begin{array}{cc}
\mathbf{A} & \mathbf{B} \\
-\mathbf{B}^{T} & H
\end{array}\right]\left\{\begin{array}{c}
\boldsymbol{\epsilon} \\
D_{z}
\end{array}\right\}
$$

where

$$
\mathbf{A}=\mathbf{C}_{=}-\mathbf{C}_{\times} \mathbf{C}_{\times}^{T} / C_{\|}, \quad \mathbf{B}=\mathbf{C}_{\times} / C_{\|} \text {and } H=1 / C_{\|} .
$$

It should be remarked that

$$
\mathbf{C}_{\times}=0 \quad \text { and } \quad C_{\|}=\infty
$$

for conductors. From (1), (14) and (15), one can obtain:

$$
\begin{aligned}
&\left\{\begin{array}{c}
\boldsymbol{\sigma}_{0} \\
\boldsymbol{\sigma}_{1} \\
\hdashline \phi_{1}-\phi_{0} \\
\vdots \\
\phi_{n}-\phi_{n-1}
\end{array}\right\}=\int_{z_{0}}^{z_{n}}\left\{\begin{array}{c}
\boldsymbol{\sigma} \\
z \boldsymbol{\sigma} \\
\hdashline \Lambda_{1} \phi_{z} \\
\vdots \\
\Lambda_{n} \phi_{z_{z}}
\end{array}\right\} d z=\int_{z_{0}}^{z_{n}}\left\{\begin{array}{c}
\mathbf{A}\left(\boldsymbol{\epsilon}_{0}+z \boldsymbol{\epsilon}_{1}\right)+\mathbf{B}\left(\Lambda_{1} D_{1}+\cdots+\Lambda_{n} D_{n}\right) \\
z \mathbf{A}\left(\boldsymbol{\epsilon}_{0}+z \boldsymbol{\epsilon}_{1}\right)+z \mathbf{B}\left(\Lambda_{1} D_{1}+\cdots+\Lambda_{n} D_{n}\right) \\
-\Lambda_{1} \mathbf{B}^{T}\left(\boldsymbol{\epsilon}_{0}+z \boldsymbol{\epsilon}_{1}\right)+\Lambda_{1} H D_{1} \\
\vdots \\
-\Lambda_{n} \mathbf{B}^{T}\left(\boldsymbol{\epsilon}_{0}+z \boldsymbol{\epsilon}_{1}\right)+\Lambda_{n} H D_{n}
\end{array}\right\} d z \\
&=\left[\begin{array}{c:c}
\tilde{\mathbf{A}} & \tilde{\mathbf{B}} \\
\hdashline-\widetilde{\mathbf{B}}^{T} & \tilde{\mathbf{H}}
\end{array}\right] \cdot\left\{\begin{array}{c}
\boldsymbol{\epsilon}_{0} \\
\boldsymbol{\epsilon}_{1} \\
D_{1} \\
\vdots \\
D_{n}
\end{array}\right\}
\end{aligned}
$$

where

$$
\begin{aligned}
\tilde{\mathbf{A}} & =\int_{z_{0}}^{z_{n}}\left[\begin{array}{cc}
\mathbf{A} & z \mathbf{A} \\
z \mathbf{A} & z^{2} \mathbf{A}
\end{array}\right] d z, \tilde{\mathbf{B}}=\int_{z_{0}}^{z_{n}}\left[\begin{array}{ccc}
\Lambda_{1} \mathbf{B} & \cdots & \Lambda_{n} \mathbf{B} \\
z \Lambda_{1} \mathbf{B} & \cdots & z \Lambda_{n} \mathbf{B}
\end{array}\right] d z, \\
\tilde{\mathbf{H}} & =\int_{z_{0}}^{z_{n}} \operatorname{diag} \cdot\left[\Lambda_{1} H, \ldots, \Lambda_{n} H\right] d z
\end{aligned}
$$

By changing equation objects, (17) can be rearranged as:

$$
\left\{\begin{array}{c}
\boldsymbol{\sigma}_{0} \\
\boldsymbol{\sigma}_{1} \\
\hdashline D_{1} \\
\vdots \\
D_{n}
\end{array}\right\}=\mathbf{C}\left\{\begin{array}{c}
\boldsymbol{\epsilon}_{0} \\
\boldsymbol{\epsilon}_{1} \\
\hdashline \phi_{1}-\phi_{0} \\
\vdots \\
\phi_{n}-\phi_{n-1}
\end{array}\right\}=\left[\begin{array}{cc}
\tilde{\mathbf{A}}+\tilde{\mathbf{B}} \tilde{\mathbf{H}}^{-1} \tilde{\mathbf{B}}^{T} & \tilde{\mathbf{B}} \tilde{\mathbf{H}}^{-1} \\
\tilde{\mathbf{H}}^{-1} \tilde{\mathbf{B}}^{T} & \tilde{\mathbf{H}}^{-1}
\end{array}\right]\left\{\begin{array}{c}
\boldsymbol{\epsilon}_{0} \\
\boldsymbol{\epsilon}_{1} \\
\hdashline \phi_{1}-\phi_{0} \\
\vdots \\
\phi_{n}-\phi_{n-1}
\end{array}\right\}
$$

where $\mathbf{C}$ is self-defined. For conductive plies sandwiched by two consecutive gauge levels, the equal-potential condition can be realized by deleting the pertinent row(s) and column(s) in C. By invoking (14), the electromechanical energy per unit planar area of the plate can be manipulated as: 


$$
\begin{aligned}
\frac{\partial U}{\partial A}= & \left.\frac{1}{2} \int_{z_{0}}^{z_{n}}\left\{\begin{array}{c}
\boldsymbol{\epsilon} \\
\phi, g_{z}
\end{array}\right\}^{T}\left\{\begin{array}{c}
\boldsymbol{\sigma} \\
D_{z}
\end{array}\right\}+\left\{\begin{array}{c}
\boldsymbol{\gamma} \\
\boldsymbol{\phi}_{L}
\end{array}\right\}^{T}\left\{\begin{array}{c}
\boldsymbol{\tau} \\
\mathbf{D}_{L}
\end{array}\right\}\right) d z \\
& =\frac{1}{2} \int_{z_{0}}^{z_{n}}\left(\left\{\begin{array}{c}
\boldsymbol{\epsilon}_{0}+z \boldsymbol{\epsilon}_{1} \\
\phi_{g_{z}}
\end{array}\right\}^{T}\left\{\begin{array}{c}
\boldsymbol{\sigma} \\
\Lambda_{1} D_{1}+\cdots+\Lambda_{n} D_{n}
\end{array}\right\}+\left\{\begin{array}{c}
\boldsymbol{\gamma} \\
\boldsymbol{\phi}_{L}
\end{array}\right\}^{T}\left\{\begin{array}{c}
\boldsymbol{\tau} \\
\mathbf{D}_{L}
\end{array}\right\}\right) d z \\
& =\frac{1}{2}\left\{\begin{array}{c}
\boldsymbol{\epsilon}_{0} \\
\boldsymbol{\epsilon}_{1}-\phi_{0} \\
\vdots \\
\phi_{n}-\phi_{n-1}
\end{array}\right\}^{T} \mathbf{C}\left\{\begin{array}{c}
\boldsymbol{\epsilon}_{0} \\
\boldsymbol{\epsilon}_{1} \\
\phi_{1}-\phi_{0} \\
\vdots \\
\phi_{n}-\phi_{n-1}
\end{array}\right\}+\frac{1}{2} \int_{z_{0}}^{z_{n}}\left(\left\{\begin{array}{c}
\boldsymbol{\gamma} \\
\boldsymbol{\phi}_{L}
\end{array}\right\}^{T}\left[\begin{array}{cc}
\mathbf{c}_{t} & \mathbf{e}_{t} \\
\mathbf{e}_{t}^{T} & -\boldsymbol{\kappa}_{t}
\end{array}\right]\left\{\begin{array}{c}
\boldsymbol{\gamma} \\
\boldsymbol{\phi}_{L}
\end{array}\right\}\right) d z
\end{aligned}
$$

Finally, the in-plane derivatives of the electric potential or $\boldsymbol{\phi}_{L}$ are computed by taking the derivatives of the piecewise linear interpolated $\phi$ at successive gauge levels as in the EC model (see (6)). Again, the problem can be computationally pursued by the Ritz method and the electromechanical virtual work principle (EerNisse 1967; Allik \& Hughes 1970). As the core assumption here is a piecewise Constant transverse electric Displacement along the transverse direction, it is termed as $D C$.

Some researchers chose to ignore the in-plane derivative of the electric potential (Almajia 2001, Lammering 1991; Trindade et al 2001). The practice is sensible by virtue of the estimated ratio in (7). As a result, (4) can be simplified to:

$$
\frac{\partial U^{*}}{\partial A}=\frac{1}{2} \int_{z_{0}}^{z_{n}}\left(\left\{\begin{array}{c}
\boldsymbol{\epsilon} \\
\phi,_{z}
\end{array}\right\}^{T}\left[\begin{array}{cc}
\mathbf{C}_{\bar{z}} & \mathbf{C}_{\times} \\
\mathbf{C}_{\times}^{T} & C_{\|}
\end{array}\right]\left\{\begin{array}{c}
\boldsymbol{\epsilon} \\
\phi,_{z}
\end{array}\right\}+\boldsymbol{\gamma}^{T} \mathbf{c}_{t} \boldsymbol{\gamma}\right) d z
$$

By adopting the same simplification, (19) becomes:

$$
\frac{\partial U^{*}}{\partial A}=\frac{1}{2}\left\{\begin{array}{c}
\boldsymbol{\epsilon}_{0} \\
\boldsymbol{\epsilon}_{1} \\
\phi_{1}-\phi_{0} \\
\vdots \\
\phi_{n}-\phi_{n-1}
\end{array}\right\}^{T} \mathbf{C}\left\{\begin{array}{c}
\boldsymbol{\epsilon}_{0} \\
\boldsymbol{\epsilon}_{1} \\
\phi_{1}-\phi_{0} \\
\vdots \\
\phi_{n}-\phi_{n-1}
\end{array}\right\}+\frac{1}{2} \int_{z_{0}}^{z_{n}} \boldsymbol{\gamma}^{T} \mathbf{c}_{t} \boldsymbol{\gamma} d z
$$

in which the variation of the electric potential along the transverse direction is no more required.

\section{Examples on Laminated Piezoelectric Beams with Conductive Electrodes}

In this section, simply-supported piezoelectric laminated beams with conductive electrodes will be attempted by using EC, EL and DC presented in the previous sections. Aspect ratios of the beams 
are chosen to be 10 which is typical for moderately thick beams. Ritz method will be employed in the electromechanical virtual work principle (EerNisse 1967, Allik \& Hughes 1970) and the trial solutions of $u_{i}$ 's and $\phi$ are polynomials of the longitudinal coordinate $x$. Again, the number of electric gauge levels is denoted by $n$. The piezoelectrics being considered is PZT-4 due to its popularity. When SI units are used to express its constitutive coefficients, the ratio of the most extreme non-zero coefficients can be as large as $10^{20}$ which may cause considerable round-off errors in 8-byte or double precision computation (Sze et al 2001). To avoid the error, the default units used for length, force, stress, charge, electric displacement and electric potential are taken to be $\mathrm{mm}, \mathrm{N}, \mathrm{N} / \mathrm{mm}^{2}, \mathrm{pC}, \mathrm{pC} / \mathrm{mm}^{2}$ and $\mathrm{GV}$, respectively. In this light, the non-zero constitutive coefficients of PZT-4 are (Park \& Sun 1995):

$$
\begin{aligned}
& c_{11}=c_{22}=139 \times 10^{3}, c_{33}=113 \times 10^{3}, c_{44}=c_{55}=25.6 \times 10^{3}, c_{66}=30.6 \times 10^{3}, \\
& c_{12}=77.8 \times 10^{3}, c_{13}=c_{23}=74.3 \times 10^{3}\left(\text { in } \mathrm{N} / \mathrm{mm}^{2}\right) ; \\
& e_{15}=e_{24}=13.44 \times 10^{6}, \mathrm{e}_{31}=\mathrm{e}_{32}=-6.98 \times 10^{6}, \mathrm{e}_{33}=13.84 \times 10^{6}\left(\text { in } \mathrm{pC} / \mathrm{mm}^{2}\right) ; \\
& \kappa_{11}=\kappa_{22}=6.00 \times 10^{9}, \kappa_{33}=5.47 \times 10^{9}(\text { in } \mathrm{pC} /(\mathrm{GVmm})) .
\end{aligned}
$$

To reduce the constitutive relations for plates in (3) to that for beams, the following generalized piezoelectric plane strain conditions are imposed:

$$
u, y=v,_{y}=\phi,,_{y}=0
$$

with which

$$
\left\{\begin{array}{l}
\sigma_{x} \\
D_{z}
\end{array}\right\}=\left[\begin{array}{cc}
c_{11}-c_{13}^{2} / c_{33} & e_{31}-c_{13} e_{33} / c_{33} \\
e_{31}-c_{13} e_{33} / c_{33} & -\kappa_{33}-e_{33}^{2} / c_{33}
\end{array}\right]\left\{\begin{array}{c}
\varepsilon_{x} \\
\phi,_{z}
\end{array}\right\},\left\{\begin{array}{c}
\tau_{z x} \\
D_{x}
\end{array}\right\}=\left[\begin{array}{cc}
c_{44} & e_{15} \\
e_{15} & -\kappa_{11}
\end{array}\right]\left\{\begin{array}{l}
\gamma_{z x} \\
\phi,_{x}
\end{array}\right\}
$$

As the supporting and electromechanical loading conditions to be considered are symmetric with respect to $x$, the following displacement trial solution can be assumed:

$$
\left\{\begin{array}{l}
w \\
u_{0} \\
u_{1}
\end{array}\right\}=\left[\begin{array}{cc:cc:c:cc}
\left(1-\xi^{2}\right) & \mathbf{0}_{1 \times 2} & \left(1-\xi^{2}\right) \xi^{2} & \mathbf{0}_{1 \times 2} & \cdots & \left(1-\xi^{2}\right) \xi^{2 m} & \mathbf{0}_{1 \times 2} \\
\mathbf{0}_{2 \times 1} & \mathbf{I}_{2} \xi & \mathbf{0}_{2 \times 1} & \mathbf{I}_{2} \xi^{3} & \cdots & \mathbf{0}_{2 \times 1} & \mathbf{I}_{2} \xi^{2 m+1}
\end{array}\right]\left\{\begin{array}{c}
\boldsymbol{\beta}_{0} \\
\boldsymbol{\beta}_{1} \\
\vdots \\
\boldsymbol{\beta}_{m}
\end{array}\right\}
$$

where $\xi=x / a, a$ is the half length of the beam, $\mathbf{I}_{2}$ is the second order identity matrix and $\boldsymbol{\beta}$ 's are vectors of coefficients. Besides the uniform electric potentials at the electrodes, the electric potential at the remaining gauge levels $\phi_{j}$ 's and all electric potential enhancement $\phi_{b}^{j}$ 's employed in 
the EL (see (11)) are taken to be:

$$
\phi_{j}=\phi_{j 0}+\xi^{2} \phi_{j 1}+\xi^{4} \phi_{j 2}+\cdots+\xi^{2 m+2} \phi_{j m}, \phi_{b}^{j}=\phi_{b 0}^{j}+\xi^{2} \phi_{b 1}^{j}+\xi^{4} \phi_{b 2}^{j}+\cdots+\xi^{2 m+2} \phi_{b m}^{j}
$$

where $\phi_{j i}$ 's and $\phi_{b i}^{j}$ 's are coefficients to be determined. Furthermore, the $m$ 's in (24) and (25) are identical in the same computational trial and the $m$ is kept increasing until the predictions are highly converged.

Since Mindlin plate theory do not aim at capturing the localized boundary effect, only the midspan predictions are reported. The mechanical predictions include the mid-thickness deflection and in-plane stress at ply-to-ply interfaces. The electric predictions include the electric potential and transverse electric displacement at electrodes not prescribed with electric potential. The electric predictions are selected as the electrodes may be connected to voltage or charge amplifiers.

\subsection{Homogeneous Piezoelectric Beam with Two Electrodes}

The homogeneous $10 \times 1 \mathrm{~mm}$ piezoelectric simply supported beam with electrodes at $z= \pm h / 2$ is subjected to end bending moments, see Figure 2a. With the lower electrode earthed, the analytical solution can be derived to be:

$$
u=-S_{11} z x, \quad w=\frac{s_{11}}{2}\left(x^{2}-a^{2}\right)-\frac{s_{13}}{2} z^{2}, \phi=\frac{g_{31}}{2}\left(\frac{h^{2}}{4}-z^{2}\right)
$$

where

$$
\left[\begin{array}{ccc}
s_{11} & s_{13} & g_{31} \\
s_{13} & s_{33} & g_{33} \\
g_{31} & g_{33} & -f_{33}
\end{array}\right]=\left[\begin{array}{ccc}
c_{11} & c_{13} & e_{31} \\
c_{13} & c_{33} & e_{33} \\
e_{31} & e_{33} & -\kappa_{33}
\end{array}\right]^{-1}
$$

For the given constitutive coefficients of PZT-4,

$$
\begin{aligned}
& s_{11}=7.922 \times 10^{-6}, s_{13}=-3.031 \times 10^{-6}, s_{33}=7.916 \times 10^{-6}\left(\mathrm{in} \mathrm{mm}^{2} / \mathrm{N}\right) \\
& g_{31}=-17.78 \times 10^{-9}, g_{33}=23.90 \times 10^{-9}\left(\mathrm{in} \mathrm{mm}^{2} / \mathrm{pC}\right) ; f_{33}=99.67 \times 10^{-12}(\mathrm{in} \mathrm{GVmm} / \mathrm{pC}) .
\end{aligned}
$$

From the analytical solution and the constitutive relation, the stress, electric displacement, resultant axial force $F$ and bending moment $M$ are:

$$
\sigma_{x}=-z, \sigma_{z}=\tau_{z x}=D_{y}=D_{z}=0, F=\int_{-h / 2}^{+h / 2} \sigma_{x} d z=0, M=\int_{-h / 2}^{+h / 2} z \sigma_{x} d z=-\frac{h^{3}}{12}
$$

Table 1 shows the midspan predictions with two gauge levels or $n=2$. Both EL and DC reproduce the exact solution. EC is erroneous in both the deflection and electric displacement. 
Table 1. Midspan predictions for homogeneous piezoelectric beam subjected to end bending.

\begin{tabular}{|c|c|c|c|c|}
\hline & EC $(n=2)$ & EL $(n=2)$ & DC $(n=2)$ & Analytical \\
\hline$\left.w\right|_{z=0} \times 10^{6}$ & -138.7 & -99.02 & -99.02 & -99.02 \\
\hline$\left.\phi\right|_{z=z_{A}}$ & 0.000 & 0.000 & 0.000 & 0.000 \\
\hline$\left.D_{z}\right|_{z=z_{A}}$ & 89.19 & 0.000 & 0.000 & 0.000 \\
\hline$\left.\sigma_{x}\right|_{z=z_{A}},-\left.\sigma_{x}\right|_{z=z_{B}}$ & -0.5000 & -0.5000 & -0.5000 & -0.5000 \\
\hline
\end{tabular}

\subsection{Bimorph Beam with Two Electrodes}

In this example, a $10 \times 1 \mathrm{~mm}$ simply supported bimorph beam is considered, see Figure $2 \mathrm{a}$. The upper and lower halves of the bimorph are poled upward and downward, respectively. Through the electrodes at $z= \pm h / 2$, the equal-potential conditions $\left.\phi\right|_{z= \pm h / 2}= \pm 10^{-7} G V$ are prescribed. By ignoring the localized boundary effect, the analytical solution can be derived to be:

$$
\begin{aligned}
& u=\frac{-24 s_{11} g_{31} z x}{h^{2}\left(4 s_{11} f_{33}+g_{31}^{2}\right)} \times 10^{-7}, \phi=\frac{4 g_{31}^{2}[2 h-3 z \cdot \operatorname{sgn}(z)] z+8 s_{11} f_{33} h z}{h^{2}\left(4 s_{11} f_{33}+g_{31}^{2}\right)} \times 10^{-7}, \\
& w=\frac{12 g_{31}\left[s_{11}\left(x^{2}-a^{2}\right)-s_{13} z^{2}\right]+8 z h\left(s_{13} g_{31}-s_{11} g_{33}\right) \cdot \operatorname{sgn}(z)}{h^{2}\left(4 s_{11} f_{33}+g_{31}^{2}\right)} \times 10^{-7}
\end{aligned}
$$

where $\operatorname{sgn}(z)$ returns the sign of $z$. From the solution and the constitutive relation,

$$
\begin{aligned}
& \sigma_{x}=\frac{8[h \cdot \operatorname{sgn}(z)-3 z] g_{31}}{h\left(4 s_{11} f_{33}+g_{31}^{2}\right)} \times 10^{-7}, \sigma_{z}=\tau_{z x}=D_{x}=0, D_{z}=\frac{-8 s_{11}}{h\left(4 s_{11} f_{33}+g_{31}^{2}\right)} \times 10^{-7}, \\
& F=\int_{-h / 2}^{+h / 2} \sigma_{x} d z=0, \quad M=\int_{-h / 2}^{+h / 2} z \sigma_{x} d z=0 .
\end{aligned}
$$

The midspan predictions are listed in Table 2 for $n=2$. Since the predicted quadratic $z$-terms of the electric potential in EL (see (11)) is zero, the electric potential yielded by EC and EL are identical. Despite of the piecewise quadratic distribution of the electric potential, DC reproduces the exact solution. EL can also reproduce the exact solution if an additional gauge level is set at $z=0$.

Table 2. Midspan predictions for bimorph (2 electrodes) under electric loading.

\begin{tabular}{|c|c|c|c|c|}
\hline & $\mathrm{EC}(n=2)$ & $\mathrm{EL}(n=2)$ & $\mathrm{DC}(n=2)$ & Analytical \\
\hline$\left.w\right|_{z=0} \times 10^{3}$ & 1.338 & 1.338 & 1.216 & 1.216 \\
\hline$\left.\sigma_{x}\right|_{z=z_{A}},-\left.\sigma_{x}\right|_{z=z_{B}}$ & 1.608 & 1.608 & 2.047 & 2.047 \\
\hline$\left.\sigma_{x}\right|_{z=0^{+}},-\left.\sigma_{x}\right|_{z=0^{-}}$ & -3.216 & -3.216 & -4.094 & -4.094 \\
\hline
\end{tabular}




\subsection{Bimorph Beam with Three Electrodes}

In this problem, a differently configured $10 \times 1 \mathrm{~mm}$ bimorph beam is considered. Besides the electrodes at $z= \pm h / 2$, there is also an electrode at $z=0$. Moreover, both the upper and lower halves of the bimorph is poled upward. The following equal-potential conditions are prescribed:

$$
\left.\phi\right|_{z=+h / 2}=\left.\phi\right|_{z=-h / 2}=10^{-7} G V \quad \text { and }\left.\quad \phi\right|_{z=0}=0 .
$$

By ignoring the localized boundary effect, the analytical solution is derived as:

$$
\begin{aligned}
& u=\frac{-24 s_{11} g_{31} z x}{h^{2}\left(4 s_{11} f_{33}+g_{31}^{2}\right)} \times 10^{-7}, \phi=\frac{8\left(s_{11} f_{33}+g_{31}^{2}\right) z \cdot \operatorname{sgn}(z)-12 g_{31}^{2} z^{2}}{h^{2}\left(4 s_{11} f_{33}+g_{31}^{2}\right)} \times 10^{-7}, \\
& w=\frac{12 g_{31}\left[s_{11}\left(x^{2}-a^{2}\right)-s_{13} z^{2}\right]+8 z h\left(s_{13} g_{31}-s_{11} g_{33}\right) \cdot \operatorname{sgn}(z)}{h^{2}\left(4 s_{11} f_{33}+g_{31}^{2}\right)} \times 10^{-7}
\end{aligned}
$$

from which and the constitutive relation,

$$
\begin{aligned}
& \sigma_{x}=\frac{8[h \cdot \operatorname{sgn}(z)-3 z] g_{31}}{h\left(4 s_{11} f_{33}+g_{31}^{2}\right)} \times 10^{-7}, \sigma_{z}=\tau_{z x}=D_{x}=0, D_{z}=\frac{-8 s_{11} \cdot \operatorname{sgn}(z)}{h\left(4 s_{11} f_{33}+g_{31}^{2}\right)} \times 10^{-7}, \\
& F=\int_{-h / 2}^{+h / 2} \sigma_{x} d z=0, M=\int_{-h / 2}^{+h / 2} z \sigma_{x} d z=0 .
\end{aligned}
$$

Compared to the bimorph with two electrodes, the present bimorph produces the same actuated deflection for only half of the electric potential difference. It can be seen in Table 3 that all the analytical midspan solutions and numerical solutions of EC and DC are the same as that in Table 2. With three gauge levels, the predictions of both EL and DC are exact. EC is erroneous.

Table 3. Midspan predictions for bimorph (3 electrodes) under electric loading.

\begin{tabular}{|c|c|c|c|c|}
\hline & $\mathrm{EC}(n=3)$ & $\mathrm{EL}(n=3)$ & $\mathrm{DC}(n=3)$ & Analytical \\
\hline$\left.w\right|_{z=0} \times 10^{3}$ & 1.338 & 1.216 & 1.216 & 1.216 \\
\hline$\left.\sigma_{x}\right|_{z=z_{A}},-\left.\sigma_{x}\right|_{z=z_{B}}$ & 1.608 & 2.047 & 2.047 & 2.047 \\
\hline$\left.\sigma_{x}\right|_{z=0^{+}},-\left.\sigma_{x}\right|_{z=0^{-}}$ & -3.216 & -4.094 & -4.094 & -4.094 \\
\hline
\end{tabular}

\subsection{Functionally Graded Piezoelectric Beam}

It is well-known that stress concentrations appear at the interfaces of dissimilar materials. The major advantage of functionally graded material is that the material properties change less abruptly so as to reduce the stress concentrations. The beam to be considered here comprises four piezoelectrics plies of equal thickness, see Figure $2 \mathrm{~b}$. Its overall dimension is $100 \times 10 \mathrm{~mm}$. 
Electrodes are coated at $z=z_{A}$ and $z=z_{D}$. The upper and lower halves of the beam are respectively upward and downward poled. $\mathrm{AB}$ and $\mathrm{CD}$ are PZT-4. BO and OC have the same elastic and permittivity coefficients as PZT-4 but their piezoelectric coefficients are only one-third of that of PZT-4. Two (at $z=z_{A}$ and $z_{D}$ ) and five (at $z=z_{A}, z_{B}, 0, z_{C}$ and $z_{D}$ ) gauge levels are considered. Through the two electrodes, the following electric loading is prescribed:

$$
\left.\phi\right|_{z= \pm h / 2}= \pm 10^{-7} G V
$$

The midspan deflection and electric potential are listed in Table 4 whereas the midspan inplane stress and transverse electric displacement are portrayed in Figures 3 and 4 . The predictions are contrasted with the highly converged finite element solutions obtained by using $39 \times 16$ ABAQUS's eight-node plane piezoelectric element. After removing the electric loading, the lower electrode is earthed and beam is loaded with the following vertical distributed force:

$$
q=-0.1 \mathrm{~N} / \mathrm{mm}
$$

Noteworthily, the Mindlin plate theory does not take the transverse normal strain into account. To enable a meaningful comparison of the finite element predictions with those yielded by various plate-based electric assumptions, the above line force is dispersed as a body force in the finite element computations. This practice will be adopted in all subsequent examples. Table 5 lists the midspan deflection and electric potential whereas the midspan inplane stress and transverse electric displacement are portrayed in Figures 5 and 6.

It can be seen in the Tables 4 to 5 and Figures 3 to 6 that EC $(n=2)$ and EL $(n=2)$ are equally inaccurate whereas the predictions of DC $(n=2), \mathrm{DC}(n=5)$ and EL $(n=5)$ are all very close to the highly converged finite element solution. Furthermore, the results of Figures 4 and 6 are supportive to the constant assumption of the transverse electric displacement along the transverse direction in DC. In this example, the listed solutions of EC, EL and DC are obtained by $m=5$, see (24) and (25), using which the number of electric potential d.o.f.s including those of the electrodes are 2, 20,8,44, 2 and 20 respectively for $\mathrm{EC}(n=2), \mathrm{EC}(n=5), \mathrm{EL}(n=2), \mathrm{EL}(n=5), \mathrm{DC}(n=2)$ and DC $(n=5)$.

Table 6 lists the predictions of EC $(n=5)$ and EL $(n=5)$ for the case of mechanical loading by keeping $m=5$ for the displacement but setting $m=0$ for the electric potential. Though the number of electric potential d.o.f.s drops to 5 and 9 for respectively EC and EL, the converged solutions deviate a lot from that in Table 5. This illustrates the importance of the x-direction expansion of the electric potential in the problem. To conclude, this example clearly illustrates the computational efficiency of DC over the others. With only 2 electric potential d.o.f.s, DC can yield results equally accurate to that of EL that employs 44 electric potential d.o.f.s. 
Table 4. Midspan predictions for functionally graded beam under uniform electric loading.

\begin{tabular}{|c|c|c|c|c|c|c|c|}
\hline & \multicolumn{2}{|c|}{ EC } & \multicolumn{2}{|c|}{ EL } & \multicolumn{2}{c|}{ DC } & \multirow{2}{*}{ ABAQUS } \\
\cline { 2 - 8 } & $n=2$ & $n=5$ & $n=2$ & $n=5$ & $n=2$ & $n=5$ & \\
\hline$\left.w\right|_{z=0} \times 10^{3}$ & 1.115 & 0.8790 & 1.115 & 0.8682 & 0.8682 & 0.8682 & 0.8680 \\
\hline$\left.\phi\right|_{z=z_{B}} \times 10^{9},-\left.\phi\right|_{z=z_{c}} \times 10^{9}$ & 50.00 & 63.22 & 50.00 & 63.13 & $63.13^{*}$ & 63.13 & 63.13 \\
\hline$\left.\phi\right|_{z=0} \times 10^{9}$ & 0 & 0 & 0 & 0 & $0^{*}$ & 0 & 0 \\
\hline
\end{tabular}

* computed by integrating $\phi_{, z}$ in (15).

Table 5. Midspan predictions for functionally graded beam under uniform mechanical loading.

\begin{tabular}{|c|c|c|c|c|c|c|c|}
\hline & \multicolumn{2}{|c|}{ EC } & \multicolumn{2}{c|}{ EL } & \multicolumn{2}{c|}{ DC } & \multirow{2}{*}{ ABAQUS } \\
\cline { 2 - 7 } & $n=2$ & $n=5$ & $n=2$ & $n=5$ & $n=2$ & $n=5$ & \\
\hline$\left.w\right|_{z=0} \times 10^{3}$ & -15.20 & -13.78 & -15.20 & -13.62 & -13.68 & -13.63 & -13.82 \\
\hline$\left.\phi\right|_{z=z_{A}} \times 10^{9}$ & 470.1 & 396.5 & 470.1 & 392.3 & 392.3 & 392.3 & 388.6 \\
\hline$\left.\phi\right|_{z=z_{B}} \times 10^{9}$ & 352.6 & 170.1 & 352.6 & 168.7 & $170.5^{*}$ & 168.4 & 166.6 \\
\hline$\left.\phi\right|_{z=0} \times 10^{9}$ & 235.1 & 198.2 & 235.1 & 196.1 & $196.1^{*}$ & 196.1 & 194.3 \\
\hline$\left.\phi\right|_{z=z_{C}} \times 10^{9}$ & 117.5 & 226.4 & 117.5 & 223.5 & $221.8^{*}$ & 223.9 & 222.0 \\
\hline
\end{tabular}

* computed by integrating $\phi, z$ in (15).

Table 6. Midspan predictions for functionally graded beam under uniform mechanical loading.

\begin{tabular}{|c|c|c|c|}
\hline & EC $(n=5, \phi \neq \phi(\mathrm{x}))$ & $\operatorname{EL}(n=5, \phi \neq \phi(\mathrm{x}))$ & ABAQUS $(\phi=\phi(\mathrm{x}))$ \\
\hline$\left.w\right|_{z=0} \times 10^{3}$ & -14.28 & -14.17 & -13.82 \\
\hline$\left.\phi\right|_{z=z_{A}} \times 10^{9}$ & 396.4 & 392.3 & 388.6 \\
\hline$\left.\phi\right|_{z=z_{B}} \times 10^{9}$ & 222.7 & 220.3 & 166.6 \\
\hline$\left.\phi\right|_{z=0} \times 10^{9}$ & 198.2 & 196.1 & 194.3 \\
\hline$\left.\phi\right|_{z=z_{C}} \times 10^{9}$ & 173.8 & 172.0 & 222.0 \\
\hline
\end{tabular}

\section{Examples on Piezoelectric Piezoelectric Beams subjected to Sinusoidal Loading}

In this section, the popular simply-supported beam problems subjected to sinusoidal loads are considered (see, e.g., Lo et al 1977, Heyliger et al 1994, Chee et al 2000, Sze et al 2000, Seikh et al 2001 and Trindade et al 2001). Common to these problems, the exact displacement and electric potential solutions can be expressed as:

$$
u=U(z) \sin \frac{\pi x}{2 a}, w=W(z) \cos \frac{\pi x}{2 a} \text { and } \phi=\Phi(z) \cos \frac{\pi x}{2 a}
$$

which lead to: 


$$
\begin{aligned}
& \sigma_{x}=\left(\frac{\pi}{2 a} c_{11} U+c_{13} W^{\prime}+e_{31} \Phi^{\prime}\right) \cos \frac{\pi x}{2 a}, \sigma_{z}=\left(\frac{\pi}{2 a} c_{13} U+c_{33} W^{\prime}+e_{33} \Phi^{\prime}\right) \cos \frac{\pi x}{2 a}, \\
& \tau_{z x}=\left(c_{55} U^{\prime}-\frac{\pi}{2 a} c_{55} W-\frac{\pi}{2 b} e_{15} \Phi\right) \cos \frac{\pi x}{2 a}, D_{x}=\left(e_{15} U^{\prime}-\frac{\pi}{2 a} e_{15} W+\frac{\pi}{2 a} \kappa_{11} \Phi\right) \sin \frac{\pi x}{2 a}, \\
& D_{z}=\left(\frac{\pi}{2 a} e_{31} U+e_{33} W^{\prime}-\kappa_{33} \Phi^{\prime}\right) \cos \frac{\pi x}{2 a}
\end{aligned}
$$

Provided that the boundary conditions are admissible in (35) and (36), it is rather trivial to show that the remaining ply-to-ply interfacial, stress equilibrium and charge conservation conditions either are automatically satisfied or are ordinary differential equations of $U(z), W(z)$ and $\Phi(z)$. Though it is still not trivial to solve $U, W$ and $\Phi$ analytically, they can conveniently be solved numerically by one-dimensional finite element formulation. To this end, the employed trial solutions for $z \in\left[z_{j}, z_{j+1}\right]$ between two consecutive ply-to-ply interfaces at $z=z_{j}$ and $z_{j+1}$ will be taken as:

$$
\left\{\begin{array}{l}
U \\
W \\
\Phi
\end{array}\right\}^{j}=\frac{z_{j}-z}{z_{j}-z_{j-1}}\left\{\begin{array}{l}
U_{j-1} \\
W_{j-1} \\
\Phi_{j-1}
\end{array}\right\}+\frac{z-z_{j-1}}{z_{j}-z_{j-1}}\left\{\begin{array}{l}
U_{j} \\
W_{j} \\
\Phi_{j}
\end{array}\right\}+\frac{\left(z-z_{j-1}\right)\left(z_{j}-z\right)}{\left(z_{j}-z_{j-1}\right)^{2}}\left[\boldsymbol{\alpha}_{0}^{j}+\zeta^{j} \boldsymbol{\alpha}_{1}^{j}+\cdots+\left(\zeta^{j}\right)^{m} \boldsymbol{\alpha}_{m}^{j}\right]
$$

where $\zeta^{j}=\left(z-z_{j-1}\right) /\left(z_{j}-z_{j-1}\right) \in[0,1]$ is the normalized transverse coordinate of the $\mathrm{i}$-th ply. The first two vectors at the right hand side of the equation are nodal variables at $z=z_{j-1}$ and $z=z_{j}$ whereas $\boldsymbol{\alpha}$ 's are the coefficient vectors of the bubble modes which vanish at $z=z_{j-1}$ and $z=z_{j}$. The bubble order $m$ is increased until the predictions between two consecutive $m$ differ by less than $0.3 \%$.

With reference to parametric form of the exact solutions in (35), the trial displacement and electric potential at the gauge levels to be used in the EC, EL and DC are taken to be:

$$
u=\left(U_{0}+z U_{1}\right) \sin \frac{\pi x}{2 a}, w=W_{0} \cos \frac{\pi x}{2 a}, \phi_{j}=\Phi_{j} \cos \frac{\pi x}{2 a}
$$

where $W_{0}, U_{0}, U_{1}$ and $\Phi_{j}$ 's are scalar coefficients to be solved. Similar predictions as considered in the last section will be reported. As the in-plane stresses in the non-piezoelectric plies yielded by all electric assumptions are within $1.0 \%$ of the converged finite element solutions, they will be not be reported for conciseness.

\subsection{Homogeneous Piezoelectric Beam with Two Electric Gauge Levels}

The conductive electrodes of the homogeneous piezoelectric beam in Section 7.1 are replaced by gauge levels. With the lower level earthed, the following distributed vertical force is applied: 


$$
q=\frac{1}{10} \cos \frac{\pi x}{2 a} \mathrm{~N} / \mathrm{mm}
$$

Table 7 lists the midspan predictions under the mechanical loading $q$. The deflection predicted by EC is most erroneous. Though the percentage errors of the electric potentials predicted by EC and $\mathrm{DC}$ at $\mathrm{A}$ are large, the absolute errors compared to $29 \mathrm{~V}$ which is the approximate electric potential at $z=0$ is rather small.

Table 7. Midspan predictions for homogeneous piezoelectric beam under mechanical loading.

\begin{tabular}{|c|c|c|c|c|}
\hline & EC & EL & DC & f.e. $(m=2)$ \\
\hline$\left.w\right|_{z=0} \times 10^{3}$ & 1.405 & 1.001 & 1.015 & 1.006 \\
\hline$\left.\phi\right|_{z=z_{A}} \times 10^{9}$ & 2.523 & 1.394 & 2.523 & 1.385 \\
\hline$\left.\sigma_{x}\right|_{z=z_{A}}$ & 6.079 & 6.079 & 6.079 & 6.103 \\
\hline$\left.\sigma_{x}\right|_{z=z_{B}}$ & -6.079 & -6.079 & -6.079 & -6.102 \\
\hline
\end{tabular}

\subsection{Bimorph Beam with Two Gauge Levels}

The two electrodes in the bimorph beam in Section 7.2 are replaced by two gauge levels which are prescribed with:

$$
\left.\phi\right|_{z= \pm h / 2}= \pm 10^{-7} \cos \frac{\pi x}{2 a} \mathrm{GV}
$$

The computed results are listed in Table 8. After removing the electric loading, the lower level is earthed and the beam is subjected to the mechanical loading stipulated in (39). Table 9 list the midspan predictions. DC is most accurate. Same as Section 7.2, the predictions of EL will be very close to that of DC if an addition gauge level is set at $z=0$.

Table 8. Midspan predictions for bimorph (2 gauge levels) subjected to electric loading.

\begin{tabular}{|c|c|c|c|c|}
\hline & EC & EL & DC & f.e. $(m=2)$ \\
\hline$\left.w\right|_{z=0} \times 10^{3}$ & 1.508 & 1.508 & 0.9595 & 0.9727 \\
\hline$\left.\sigma_{x}\right|_{z=z_{A}},-\left.\sigma_{x}\right|_{z=z_{B}}$ & 1.608 & 1.608 & 2.047 & 2.032 \\
\hline$\left.\sigma_{x}\right|_{z=0^{+}},-\left.\sigma_{x}\right|_{z=0^{-}}$ & -3.216 & -3.216 & -4.094 & -4.068 \\
\hline
\end{tabular}


Table 9. Midspan predictions for bimorph (2 gauge levels) subjected to mechanical loading.

\begin{tabular}{|c|c|c|c|c|}
\hline & EC & EL & DC & f.e. $(m=2)$ \\
\hline$\left.w\right|_{z=0} \times 10^{3}$ & 1.113 & 1.113 & 1.036 & 1.039 \\
\hline$\left.\phi\right|_{Z=Z_{A}} \times 10^{9}$ & -55.36 & -55.36 & -51.25 & 50.40 \\
\hline$\left.\sigma_{x}\right|_{z=z_{A}}$ & 5.634 & 5.641 & 6.108 & 6.133 \\
\hline$\left.\sigma_{x}\right|_{z=0^{+}}$ & 0.8902 & 0.8832 & -0.05716 & -0.08370 \\
\hline$\left.\sigma_{x}\right|_{z=0^{-}}$ & -0.8902 & -0.8973 & 0.05716 & 0.1288 \\
\hline$\left.\sigma_{x}\right|_{z=z_{B}}$ & -5.634 & -5.627 & -6.108 & -6.178 \\
\hline
\end{tabular}

\subsection{Bimorph with Three Gauge Levels}

The three electrodes in the bimorph beam in Section 7.3 are replaced by three gauge levels and the middle one is earthed. The other two levels are prescribed with:

$$
\left.\phi\right|_{z= \pm h / 2}=10^{-7} \cos \frac{\pi x}{2 a} \mathrm{GV}
$$

The computed results are listed in Table 10. After removing the electric loading, the beam is subjected to the mechanical loading given in (39). Table 11 lists the midspan predictions. EC is least accurate whereas EL and DC are very close in accuracy.

Table 10. Midspan predictions for bimorph (3 gauge levels) subjected to electric loading.

\begin{tabular}{|c|c|c|c|c|}
\hline & EC & EL & DC & f.e. $(m=2)$ \\
\hline$\left.w\right|_{z=0} \times 10^{3}$ & 1.058 & 0.9613 & 0.9595 & 0.9727 \\
\hline$\left.\sigma_{x}\right|_{z=z_{A}},-\left.\sigma_{x}\right|_{z=z_{B}}$ & 1.608 & 2.029 & 2.047 & 2.032 \\
\hline$\left.\sigma_{x}\right|_{z=0^{+}},-\left.\sigma_{x}\right|_{z=0^{-}}$ & -3.216 & -4.058 & -4.094 & -4.068 \\
\hline
\end{tabular}

Table 11. Midspan predictions for bimorph (3 gauge levels) subjected to mechanical loading.

\begin{tabular}{|c|c|c|c|c|}
\hline & EC & EL & DC & f.e. $(m=2)$ \\
\hline$\left.w\right|_{z=0} \times 10^{3}$ & 1.109 & 1.026 & 1.032 & 1.031 \\
\hline$\left.\phi\right|_{z=z_{A}} \times 10^{9}$ & -28.11 & -26.04 & -26.04 & -26.07 \\
\hline$\left.\sigma_{x}\right|_{z=z_{A}},-\left.\sigma_{x}\right|_{z=z_{B}}$ & 5.627 & 6.115 & 6.099 & 6.138 \\
\hline$\left.\sigma_{x}\right|_{z=0^{+}},-\left.\sigma_{x}\right|_{z=0^{-}}$ & 0.9042 & -0.07205 & -0.04031 & -0.07092 \\
\hline
\end{tabular}

\subsection{Two-ply Laminated Piezoelectric Beam}

Figure $2 \mathrm{c}$ shows a beam formed by bonding an upward-poled $100 \times 2 \mathrm{~mm}$ PZT-4 ply to a $100 \times 10$ mm aluminum ply. Elastic modulus and Poisson's ratio of aluminum are respectively $70 \times 10^{3}$ $\mathrm{N} / \mathrm{mm}^{2}$ and 0.35 . The aluminum is earthed and the only active gauge level is at the upper surface of 
PZT-4. The level is prescribed with:

$$
\left.\phi\right|_{Z=Z_{A}}=10^{-7} \cos \frac{\pi x}{2 a} .
$$

The computed results are listed in Table 12. After removing the above electric loading, the beam is subjected to the mechanical loading given in (39). Table 13 list the predictions. Since the thickness of the piezoelectric ply is only one-sixth of the overall thickness, EC is relative more accurate compared to itself in the previous examples. Nevertheless, EL and DC are still distinctively more accurate as seen in the stress predictions.

Table 12. Midspan predictions for two-ply beam under electric loading.

\begin{tabular}{|c|c|c|c|c|}
\hline & EC & EL & DC & f.e. $(m=2)$ \\
\hline$\left.w\right|_{z=0} \times 10^{3}$ & 0.6600 & 0.6587 & 0.6587 & 0.6649 \\
\hline$\left.\sigma_{x}\right|_{z=z_{A}}$ & 0.3074 & 0.2859 & 0.2845 & 0.2814 \\
\hline$\left.\sigma_{x}\right|_{z=z_{B}^{+}}$ & -0.4256 & -0.4482 & -0.4497 & -0.4490 \\
\hline
\end{tabular}

Table 13. Midspan predictions for two-ply beam subjected to mechanical loading.

\begin{tabular}{|c|c|c|c|c|}
\hline & EC & EL & DC & f.e. $(m=2)$ \\
\hline$\left.w\right|_{z=0} \times 10^{3}$ & 7.883 & 7.868 & 7.869 & 7.922 \\
\hline$\left.\phi\right|_{z=z_{A}} \times 10^{9}$ & -151.1 & -150.9 & -150.9 & -151.1 \\
\hline$\left.\sigma_{x}\right|_{z=z_{A}}$ & 4.950 & 5.220 & 5.210 & 5.238 \\
\hline$\left.\sigma_{x}\right|_{z=z_{B}^{+}}$ & 3.607 & 3.321 & 3.318 & 3.314 \\
\hline
\end{tabular}

\subsection{Three-ply Laminated Piezoelectric Beam}

Figure $2 \mathrm{~d}$ shows a laminated piezoelectric beam formed by bonding two upward-poled $100 \times 2$ $\mathrm{mm}$ PZT-4 plies to a $100 \times 10 \mathrm{~mm}$ aluminum. The aluminum is earthed and there are two active gauge levels at A and B. The following electric loading is prescribed:

$$
\left.\phi\right|_{z=z_{A}}=\left.\phi\right|_{z=z_{D}}=10^{-7} \cos \frac{\pi x}{2 a}
$$

The predictions are listed in Table 14. After removing the electric loading, the beam is subjected to the mechanical loading given in (39). The predictions are presented in Table 15. The thickness of the piezoelectric ply is only one-seventh of the overall thickness. EC is marginally more accurate in the deflection and electric potential than EL and DC. Nevertheless, EL and DC are still markedly more accurate in the stress predictions.

Table 14. Midspan deflections at $z=0$ for three-ply beam under electric loading. 


\begin{tabular}{|c|c|c|c|c|}
\hline & EC & EL & DC & f.e. $(m=2)$ \\
\hline$\left.w\right|_{z=0} \times 10^{3}$ & 0.9825 & 0.9801 & 0.9801 & 0.9915 \\
\hline$\left.\sigma_{x}\right|_{z=z_{A}},-\left.\sigma_{x}\right|_{z=z_{D}}$ & -0.1874 & -0.1546 & -0.1538 & -0.1516 \\
\hline$\left.\sigma_{x}\right|_{z=z_{B}^{+}},-\left.\sigma_{x}\right|_{z=z_{C}^{-}}$ & -0.3636 & -0.3989 & -.3999 & -0.3999 \\
\hline
\end{tabular}

Table 15. Midspan predictions for three-ply beam under mechanical loading.

\begin{tabular}{|c|c|c|c|c|}
\hline & EC & EL & DC & f.e. $(m=2)$ \\
\hline$\left.w\right|_{z=0} \times 10^{3}$ & 4.413 & 4.404 & 4.405 & 4.443 \\
\hline$\left.\phi\right|_{z=z_{A}} \times 10^{9},\left.\phi\right|_{z=z_{D}} \times 10^{9}$ & -108.4 & -108.2 & -108.2 & -108.6 \\
\hline$\left.\sigma_{x}\right|_{z=z_{A}},-\left.\sigma_{x}\right|_{z=z_{D}}$ & 3.441 & 3.590 & 3.581 & 3.610 \\
\hline$\left.\sigma_{x}\right|_{z=z_{B}^{+}},-\left.\sigma_{x}\right|_{z=z_{C}^{-}}$ & 2.707 & 2.545 & 2.555 & 2.542 \\
\hline
\end{tabular}

\subsection{Functionally Graded Piezoelectric Beam}

With the electrodes replaced by electric gauge levels, the functionally graded piezoelectric beam in Section 7.4 is considered. Again, two $(n=2)$ and five $(n=5)$ gauge levels are employed. The electric loading given in (42) is applied. The midspan deflection and electric potential are listed in Table 15 whereas the inplane stress and transverse electric displacement are plotted in Figures 8 and 9, respectively. After removing the electric loading, the beam is subjected to the mechanical loading given in (39). The midspan deflection and electric potential are presented in Table 16 whereas the inplane stress and transverse electric displacement are plotted in Figures 10 and 11, respectively. EL $(n=5)$ and DC $(n=5)$ are most accurate whereas EC $(n=2)$ and EL $(n=2)$ are least accurate. Despite of the smaller number of electric potential d.o.f.s, DC $(n=2)$ is only marginally less accurate than EL $(n=5)$ and DC $(n=5)$. Furthermore, the results of Figures 8 and 10 are supportive to the constant assumption of the transverse electric displacement along the transverse direction in DC.

Table 15. Midspan predictions for functionally graded beam under sinusoidal electric loading.

\begin{tabular}{|c|c|c|c|c|c|c|c|}
\hline & \multicolumn{2}{|c|}{$\mathrm{EC}$} & \multicolumn{2}{c|}{ EL } & \multicolumn{2}{c|}{ DC } & f.e. \\
\cline { 2 - 8 } & $n=2$ & $n=5$ & $n=2$ & $n=5$ & $n=2$ & $n=5$ & $(m=2)$ \\
\hline$\left.w\right|_{z=0} \times 10^{3}$ & 0.8818 & 0.6918 & 0.8818 & 0.6834 & $0.6819^{*}$ & 0.6830 & 0.6943 \\
\hline$\left.\phi\right|_{z=z_{B}} \times 10^{9},-\left.\phi\right|_{z=z_{c}} \times 10^{9}$ & 50.00 & 62.99 & 50.00 & 62.90 & $63.13^{*}$ & 62.89 & 62.89 \\
\hline$\left.\phi\right|_{z=0} \times 10^{9}$ & 0 & 0 & 0 & 0 & $0 *$ & 0 & 0 \\
\hline
\end{tabular}

* computed by integrating $\phi, z$ in (15). 
Table 16. Midspan predictions for functionally graded beam under sinusoidal mechanical loading.

\begin{tabular}{|c|c|c|c|c|c|c|c|}
\hline & \multicolumn{2}{|c|}{$\mathrm{EC}$} & \multicolumn{2}{|c|}{ EL } & \multicolumn{2}{|c|}{$\mathrm{DC}$} & \multirow{2}{*}{$\begin{array}{c}\text { f.e. } \\
(m=2)\end{array}$} \\
\hline & $n=2$ & $n=5$ & $n=2$ & $n=5$ & $n=2$ & $n=5$ & \\
\hline$\left.w\right|_{z=0} \times 10^{3}$ & 11.67 & 10.68 & 11.67 & 10.56 & 10.61 & 10.57 & 10.64 \\
\hline$\left.\phi\right|_{z=z_{A}} \times 10^{9}$ & -542.1 & -453.1 & -542.2 & -448.3 & -448.3 & -448.3 & -445.4 \\
\hline$\left.\phi\right|_{z=z_{B}} \times 10^{9}$ & -406.6 & -243.9 & -404.8 & -241.4 & $-244.5^{*}$ & -241.2 & -239.1 \\
\hline$\left.\phi\right|_{z=0} \times 10^{9}$ & -271.0 & -224.5 & -268.7 & -222.1 & $-224.2^{*}$ & -222.1 & -220.2 \\
\hline$\left.\phi\right|_{z=z_{C}} \times 10^{9}$ & -135.5 & -206.3 & -133.8 & -204.4 & $-203.8^{*}$ & -204.1 & -203.1 \\
\hline
\end{tabular}

* computed by integrating $\phi, z$ in (15).

\section{Closure}

In computational analysis of piezoelectric laminates, the transverse electric field is often assumed to be piecewise constant along the transverse direction. It is first argued that the transverse Electric field is essentially piecewise Linear along the transverse direction. Based on the argument, $E L$ is proposed. As the electric field is not continuous at the interface of dissimilar plies, each piecewise function in EL should span only one ply. It is then argued that the transverse electric Displacement is essentially Constant along the transverse direction within a stack of plies inside which there is no free charge-flow. Based on the argument, $D C$ is proposed. The analytical or highly converged finite element solutions are supportive to the argument leading to DC. As the piecewise function in DC can span more than one ply, it is computationally more efficient than EL in general.

The computational advantage of DC is most advantageous in modeling practical laminated piezoelectric plates in which the piezoelectric plies are sandwiched by conductive electrodes. In these structures, the only needed electric potential d.o.f.s in DC are the electric potential of the electrodes. To deliver similar accuracy, both the conventional assumption and EC require extra electric gauge levels set at ply-to-ply interface. The worst is that electric potential at these extra gauge levels and the electric potential enhancements in EL are functions of the in-plane coordinates. The pertinent expansion of the electric potential leads to a large number of electric potential d.o.f.s.

The computational efficiency of DC can be further improved by dropping the electromechanical arising from the in-plane components of the electric field (see (21)). With these components taken to be zero, the accuracy of DC in laminated piezoelectric plates with conductive electrodes remain essentially intact.

Acknowledgment - The financial support of Research Grant Council of Hong Kong (CERG: HKU 7082/97E) is gratefully acknowledged. 


\section{REFERENCES}

ABAQUS (2000). ABAQUS User Manual Version 6.1. Hibbitt, Karlsson \& Sorensen Inc. Rhode Island.

Allik H., Hughes T.J. R. (1970). Finite element method for piezoelectric vibration. Inter.J. Numer. Methods Engrg., 2: 151-157.

Almajia A. (2001). Analysis of out-of-plne displacement and stress field in a piezocomposite plate with functionally graded microstructure. Inter.J.Solids \& Stuctures, 38: 3377-3391.

Chee C., Tong L., Steven G. (2000). A mixed model for adaptive composite plates with piezoelectric for anisotropic acutation. Computer \&structure, 77: 253-268 .

EerNisse EP. (1967) Variational method for electroelastic vibration analysis. IEEE Trans. Sonics \& Ultrasonics, 14:153-160.

Fernandes A, Pouget J (2001). Two-dimensional modeling of laminated piezoelectric composites: analysis and numerical results. Thin-Walled Structures, 39: 3-22.

HA S.K., KeILERS C. and Chang F.K. (1992). Finite element analysis of composite structures containing distributed piezoelectric sensors and actuators. AIAA J., 30: 772-780.

Heyliger P, Ramirez G, Saravanos D (1994). Coupled discrete-layer finite elements for laminated piezoelectric plates. Comm.Numer.Methods Engrg., 10: 971-981.

Lammering R (1991). The application of a finite shell element for composites containing piezo-electric polymers in vibration control. Computers \& Structures, 41: 1101-1109.

Lo KH, Christensen RM, Wu EM (1977). A high-order theory of plate deformation - Part 1 : homogeneous plates and Part 2 : laminated plates. J.Appl.Mech.-Trans.ASME, 44: 663-676.

Noor AK, Burton WS (1989). Assessment of shear deformation theories for multilayered composite plates. Applied Mechanics Review., 42: 1-12.

Park SB, Sun CT (1995). Effect of electric field on fracture of piezoelectric ceramics. Int.J. Fracture, 70: 203-216.

Reddy JN (1984). A simple higher-order theory for laminated composite plates. J.Appl.Mech. - Trans.ASME, 51: 745-752.

Sheikh AH, Topdar P, Halder S (2001). An appropriate FE for through-thickness variation of displacement and potential in thin/moderately thick smart laminates. Composite structure, 51: 401-409 .

Sze KY, Pan YS (1999). Hybrid piezoelectric finite element models for three-dimensional analysis. J.Sound \& Vibration, 226: 519-547.

Sze KY, Yao LQ, Yi S (2000). A hybrid-stress ANS solid-shell element and its generalization for smart structure modelling - part I: solid-shell element formulation, part II: smart structure modeling. Inter.J.Numer.Methods Engrg., 48: 545-582.

Sze KY, He LW, Cheung YK (2000). Predictor-corrector procedures for analysis of laminated Plates using standard Mindlin finite element models. J.Composite Structures, 50: 171-182.

Sze KY, Wang HT, Fan H (2001). A finite element approach for computing edge singularities in piezoelectric materials. Inter.J.Solids \& Structures, 38: 9233-9252.

Trindade M.A., Benjeddou A., Ohayon R. (2001). Finite element modelling of hybrid active-passive vibration damping of multiplayer piezoelectric sandwich beams-part I: Formulation. Inter.J. Numer.Methods Engrg. 51: 835-854.

Tzou HS, Tseng CI, Bahrami H (1994). A thin piezoelectric hexahedron finite element applied to design of smart continua. Finite Elements in Analysis \& Design, 16: 27-42.

Tzou HS, Ye R (1996). Analysis of piezoelectric structures with laminated piezoelectric triangle shell elements. AIAA J., 34,110-115.

Yang JS (1999). Equations for the extension and flexure of electroelastic plates. J.Appl.Mech., 36 : 3171-3192. 


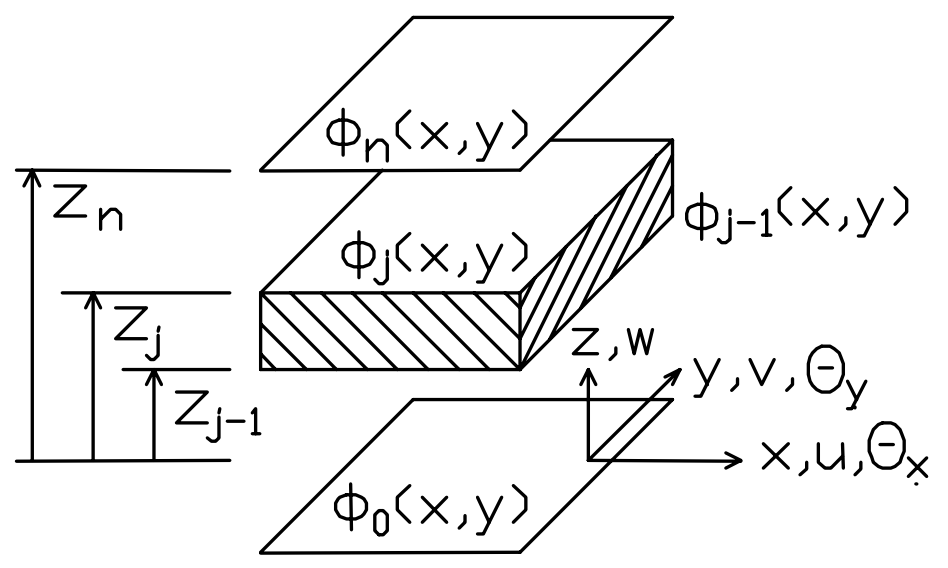

Figure 1. Electric potential at different electric gauge levels.

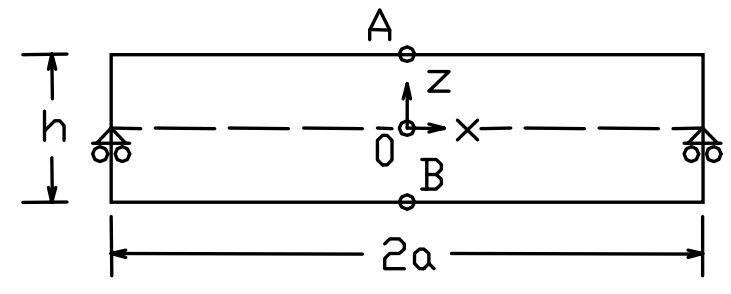

(a)

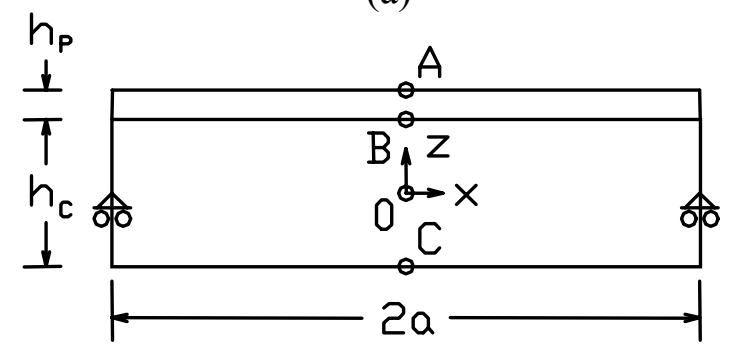

(c)

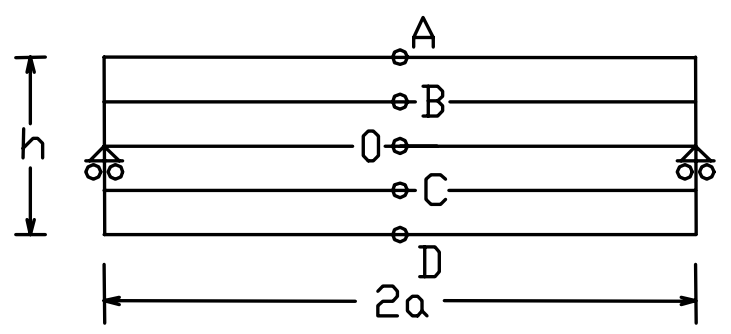

(b)

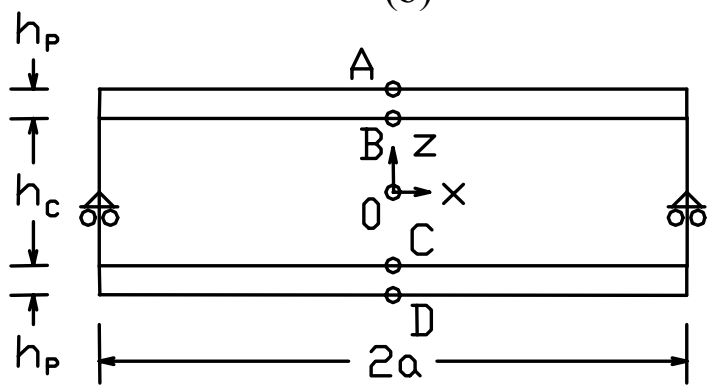

(d)

Figure 2. Simply supported (a) homogeneous/bimorph piezoelectric beam, (b) two-layered beam, (c) three-layered beam and (e) functionally graded piezoelectric beam. 

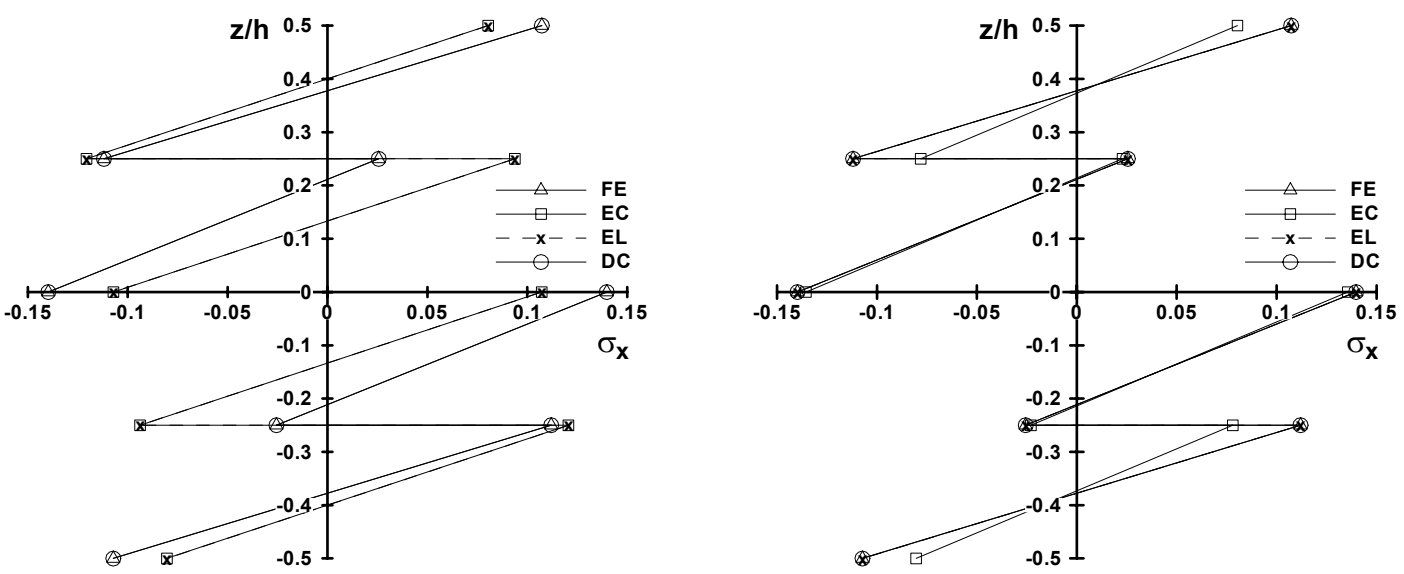

Figure 3. Inplane stress of the functionally graded piezoelectric beam under uniform electric loading modelled with (a) two and (b) five electric gauge levels.
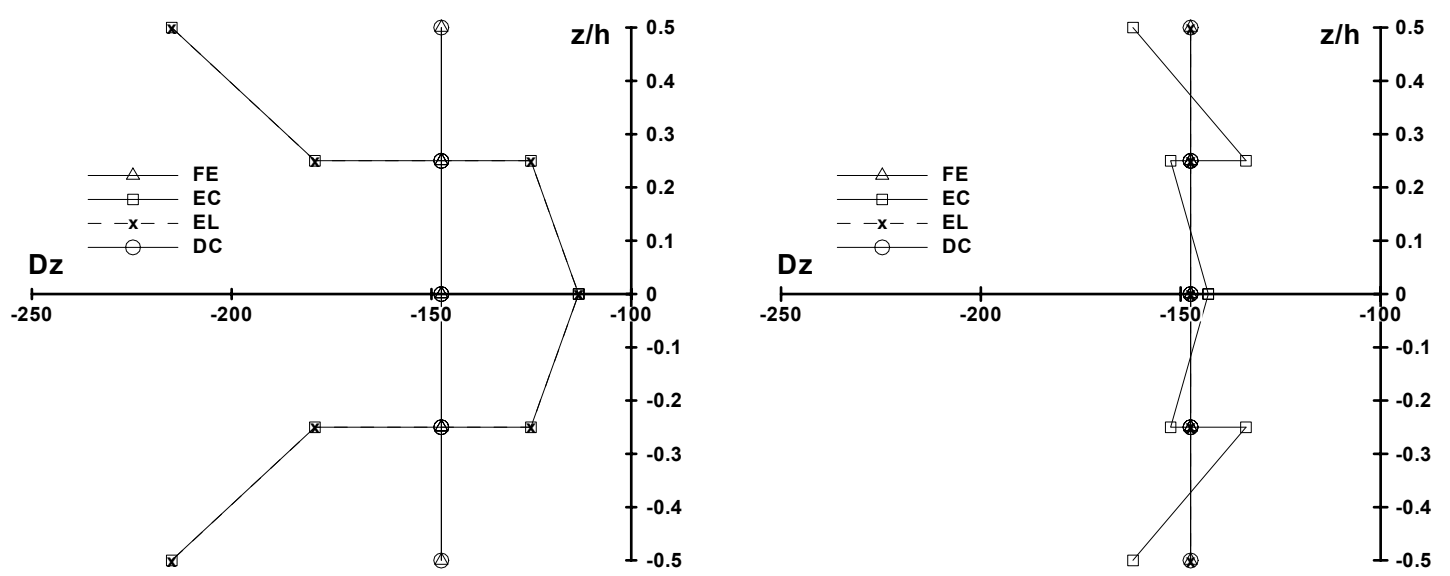

Figure 4. Transverse electric displacement of the functionally graded piezoelectric beam under uniform electric loading modelled with (a) two and (b) five electric gauge levels.
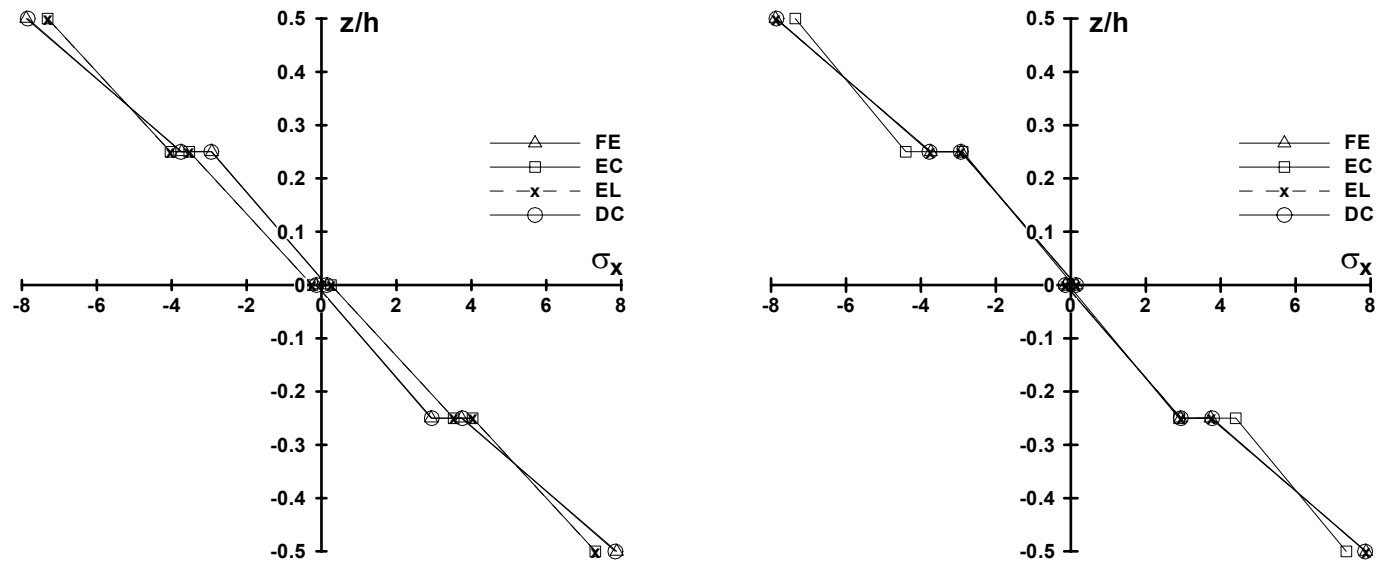

Figure 5. Inplane stress of the functionally graded piezoelectric beam under uniform mechanical loading modelled with (a) two and (b) five electric gauge levels. 

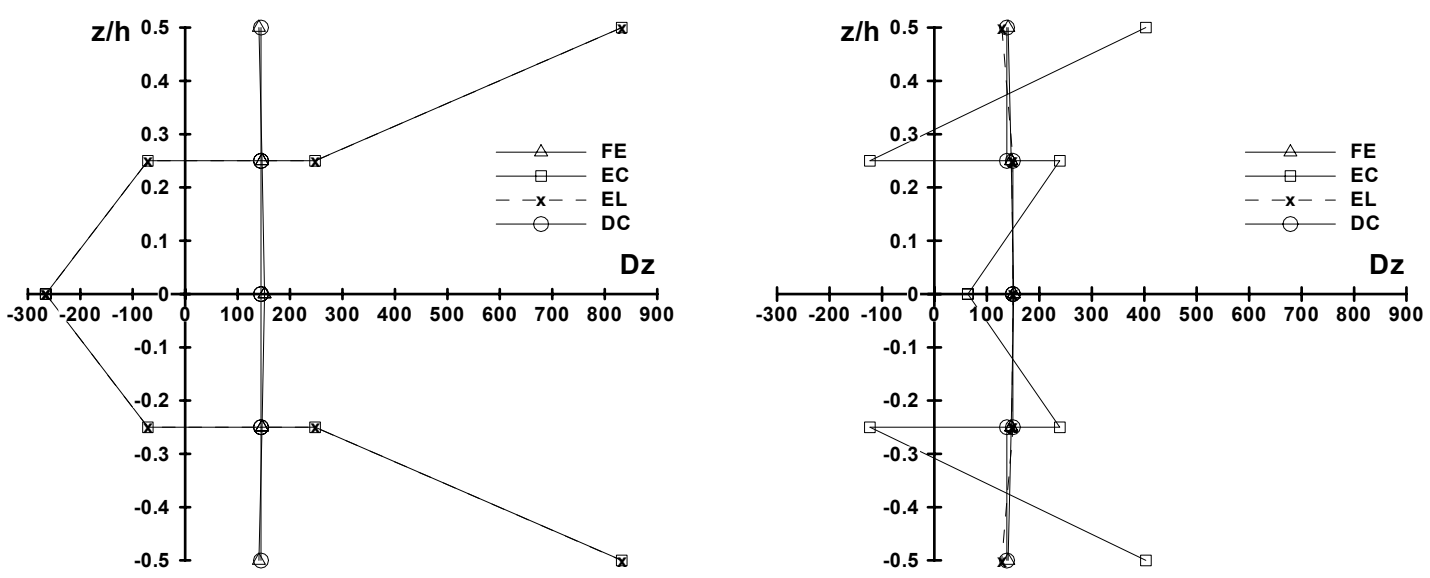

Figure 6. Transverse electric displacement of the functionally graded piezoelectric beam under uniform mechanical loading modelled with (a) two and (b) five electric gauge levels.
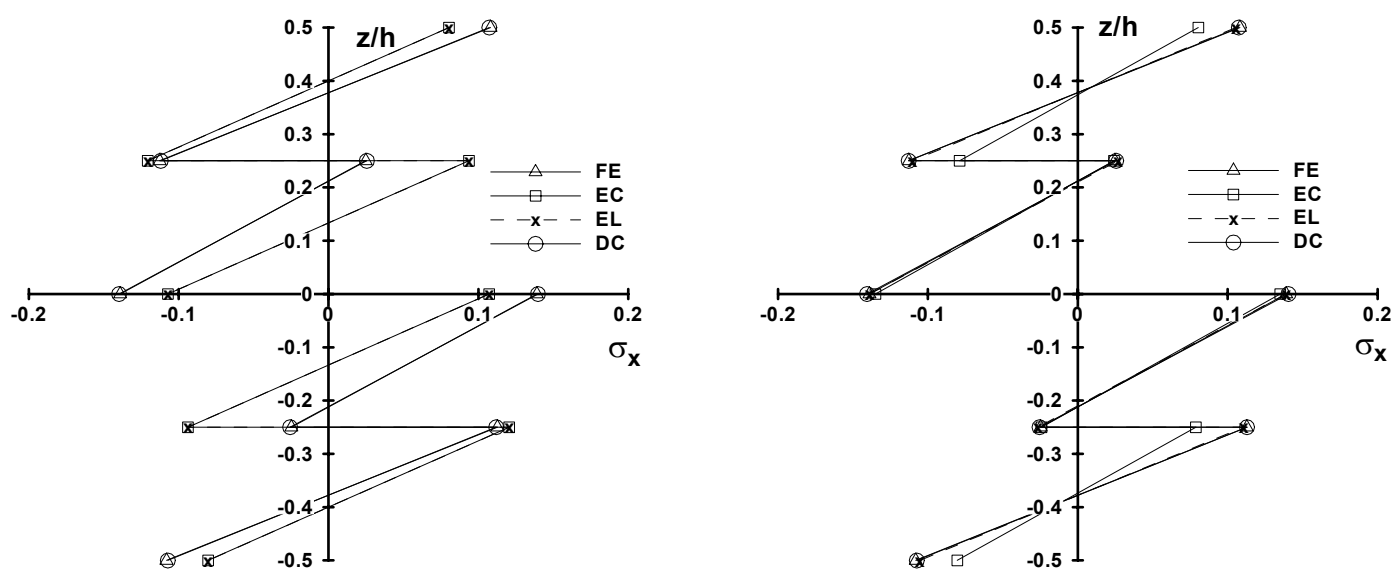

Figure 7. Inplane stress of the functionally graded piezoelectric beam under sinusoidal electric loading modelled with (a) two and (b) five electric gauge levels.
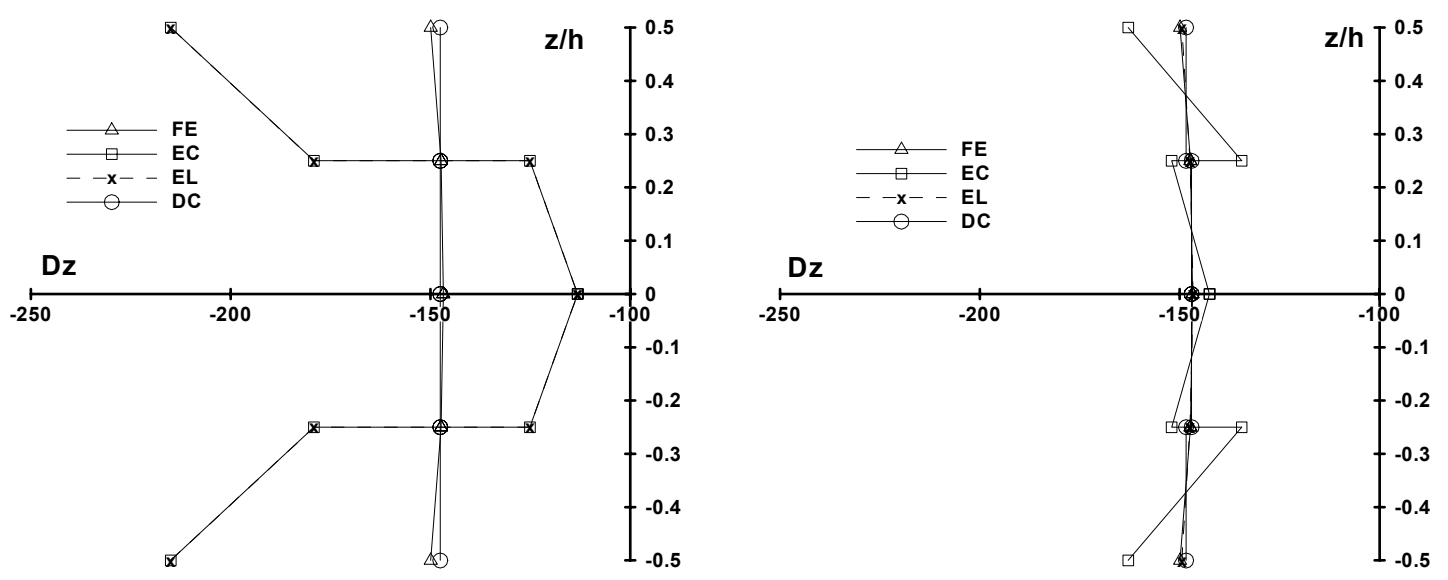

Figure 8. Transverse electric displacement of the functionally graded piezoelectric beam under sinusoidal electric loading modelled with (a) two and (b) five electric gauge levels. 

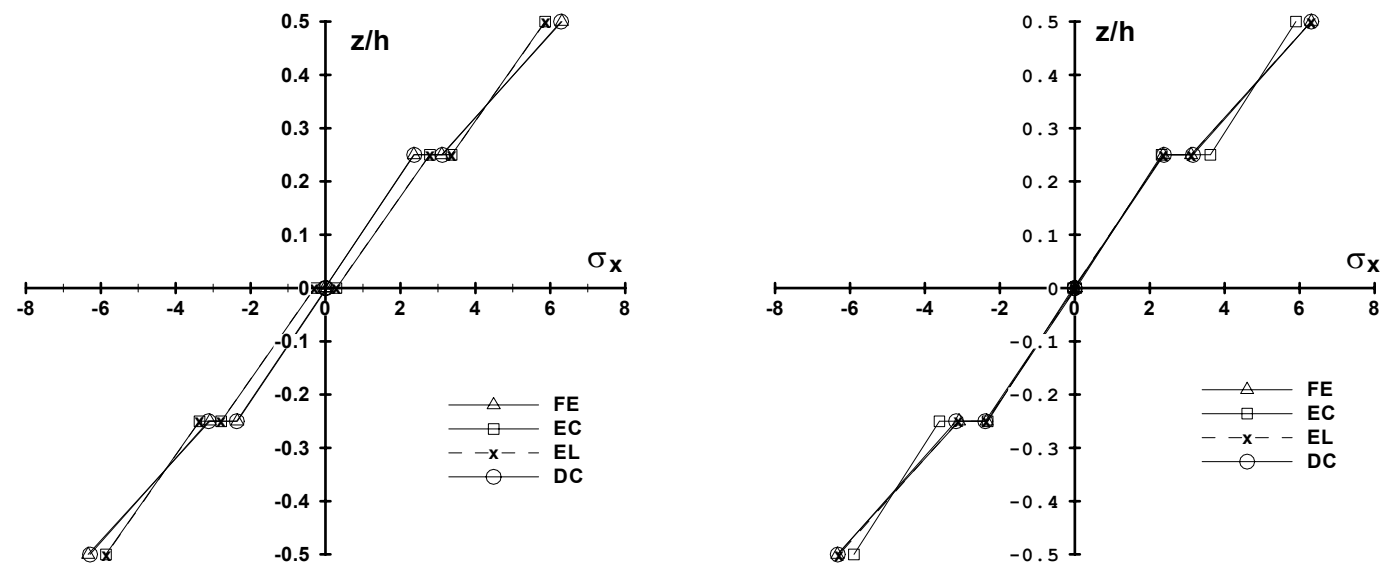

Figure 9. Inplane stress of the functionally graded piezoelectric beam under sinusoidal mechanical loading modelled with (a) two and (b) five electric gauge levels.
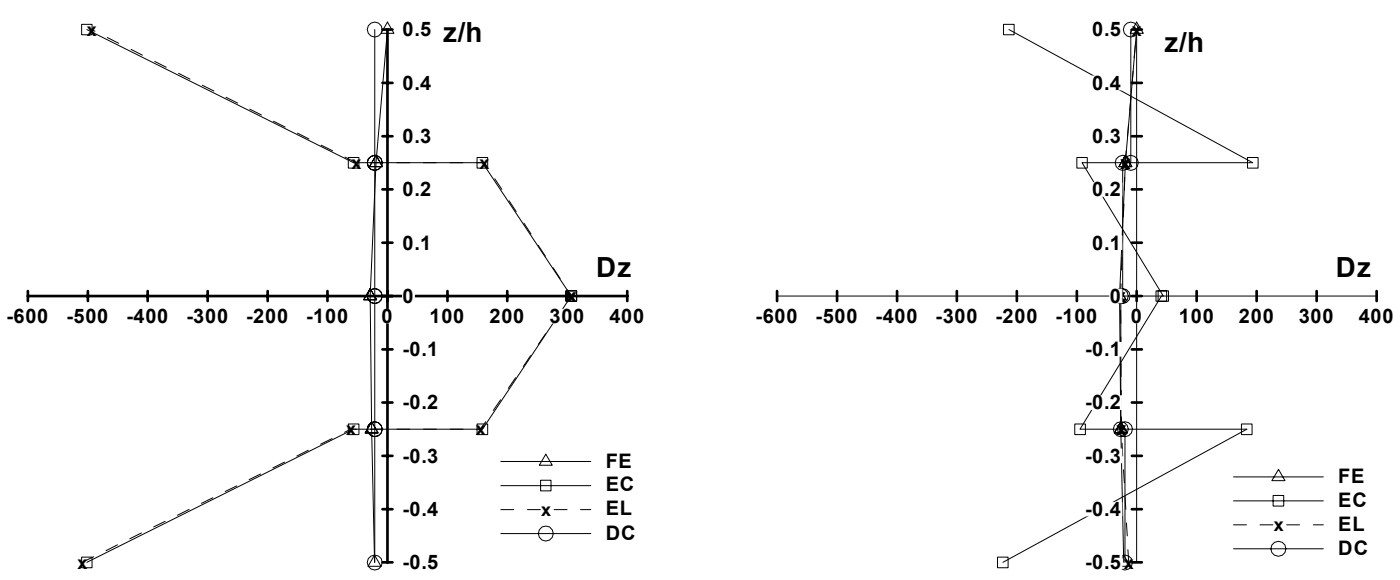

Figure 10. Transverse electric displacement of the functionally graded piezoelectric beam under sinusoidal mechanical loading modelled with (a) two and (b) five electric gauge levels. 